\title{
Creative Dance: an Approach for Social Interaction between Robots and Children
}

\author{
Raquel Ros, Yiannis Demiris \\ Imperial College London, SW7 2BT, London, UK \\ \{raquel.ros, y.demiris\}@imperial.ac.uk
}

\begin{abstract}
In this paper we discuss the potential of using a dance robot tutor with children in the context of creative dance to study child-robot interaction through several encounters. We have taken part of dance sessions in order to extract strategies and models to inspire and justify the design of a robot dance tutor. Moreover, we present implementation details and preliminary results on a pilot study to extract initial feedback to further improve and test our system with a broader children population.
\end{abstract}

Keywords: child-robot interaction, creative dance, long-term interaction, edutainment

\section{Introduction}

Dance is considered an entertaining activity that allows children to easily engage [14], probably due to the involvement of body movement during the activity which in turn increases enjoyment [2]. But more importantly it $i$ ) stimulates physical movement, which is essential for the development of fundamental motor skills; ii) enhances the development of social skills, such as cooperation, coordination, sharing ideas, sharing physical space, accepting individual differences; iii) increases communication skills; and $i v$ ) promotes the development of creativity and spontaneity [13] [10]. Considered an important educational source, dance is also promoted at schools as part of their curricula (e.g. in England it is part of the Curriculum for England in Physical Education [1]). Dance is not only a good practice for children, but for people in any age range, including elderly people where it has demonstrated to have physical and psychological benefits [7].

In this paper we discuss the potential of using a dance robot tutor with children in the context of creative dance. While our ultimate goal is to study and identify the necessary social skills required to enhance interactions between humans and robots, a more specific one and directly addressed in our current work is to study the impact of creativity in a tutoring setting throughout repeated occasions (at least three different interactions).

Within this context, we believe that in order to engage and sustain interaction between children and robots, besides providing a robotic system with basic social cues (such as head movement, eye-contact, spatial-orientational arrangement, name reference, etc.), it is essential to provide space for the user to explore 
and develop its own ideas. Moreover, children are curious and therefore, tend to be more creative than adults by nature. Stimulating and rewarding curiosity is important to enhance the development of creativity [11].

Environments where the pupils are restrained to reproduce the tutor's instructions in a specific way, such as traditional dance classes, may lead pupils to an unsatisfactory state. This risk is higher in environments where the pupil performs the activity without expecting to be an expert (in this case, professional dancers). On the contrary, exploiting creativity leads to feeling more satisfied [5], which in turn, may increase the motivation and engagement in the interaction. From our previous work [12] we have observed that during the first stages of the interaction, children felt motivated and were looking forward to continuing the task. However, as the novelty effect diminished, the task became more tedious and their engagement decreased through time. Limiting the children's spontaneity was a plausible reason for this effect.

An adequate environment can provide stimuli to express ideas and thus, develop creativity [11]. In this work, we propose to use creative dance as a mean to engage the child with the robot in a creative process to explore their own body movement, while linking these ideas to a specific theme.

\section{Creative Dance Framework Design}

In this work we refer to creative dance as a form of dance where the goal is to explore the body movement based on a set of general guidelines (movement concepts). Thus, on the one hand creative dance provides foundations on elements of dance, movement vocabulary and locomotive skills. And on the other, it promotes creativity motivating pupils to expand and extend movement range at their own rhythm through different stimuli, such as music, emotions, visual resources and observing the creations of others.

Moreover, creative dance can be used as an instrument to convey concepts that children work in their everyday school activities. Some theme examples are the states of water, creation and evolution of volcanoes, painting styles. Since our work is part of the ALIZ-E ${ }^{1}$ project, where the targeted children suffer from diabetes or obesity, a candidate knowledge area under investigation is nutrition and healthy diet. Dance concepts are linked to fruits, vegetables, simple/complex carbohydrates and proteins through the sessions. Children have an overview of what they are, how they benefit our body and how often we should eat them to achieve a healthy diet.

To study creative dance and to further design a robot dance tutor we recorded a series of dance sessions. While the global goal is to study interaction strategies used through time between a tutor and pupils, a more specific one within the dance context is to analyze dance methodologies and techniques to teach dance.

A professional dance teacher prepared a 5 -days course where the goal was to teach creative dance linked to healthy diet. Fig. 1 depicts a snapshot of one of

\footnotetext{
$\overline{{ }^{1} \text { www.aliz-e.org }}$
} 


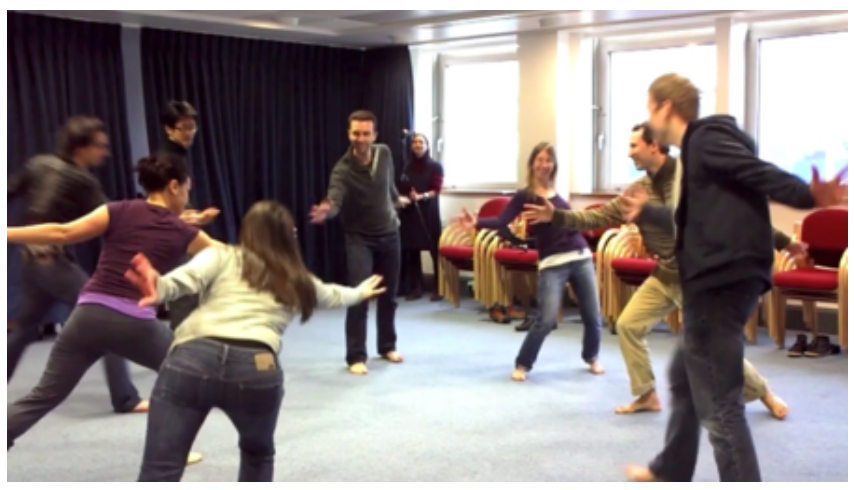

Fig. 1: Snapshot of a dance session with a professional dance teacher.

the sessions. Although the participants in the dance sessions were adults $(\mathrm{PhD}$ students from the department) to allow us to reuse the collected information in our children experiments, the teacher was instructed to perform the sessions as if the they were children between 8 and 10-years-old. This had an special impact on the instructions given, where the dance teacher used simple words and examples that a population of such age would perfectly understand. However, it did not affect neither the methodology nor the movements performed during the sessions. Each participant, of any age, can perform any of the movements within its own capabilities while learning the same foundations.

\subsection{Dance Sessions Observations}

Sessions were recorded and transcribed to extract task-based and interactionbased features to guide the design of the dance robot. We next summarize them:

- Domain-specific knowledge: describing concepts to explore is essential to start creating. If no guideline is given, hardly any new movement can emerge (or quite rarely). Providing a purpose and acquiring domain-specific knowledge is required to produce creative expression [11]. Once pupils understand the concepts, they are able to create movements based on those concepts.

- Non-verbal and verbal feedback: While the former corresponds to performing movements (or examples of the motion), the latter corresponds to providing verbal description of the concept. Both are essential to guide the pupils' comprehension and learning. Non-verbal feedback serves as visual input providing most of the information they need to understand the concepts. Verbal feedback is useful to help children recall and name the ideas worked. Analogies are powerful descriptors easy to retain to recall the concepts taught.

- User specific adaptation: The teacher constantly observes and evaluates the different capabilities of the pupils. When they manage to perform a movement or sequence of movements (either individually or in small groups) with- 
out much difficulty, she immediately asks them to go one step further challenging them. She would usually guide them increasing the complexity of the performance and then let them explore by themselves as much as they can.

- Learning resources: Additional visual resources are used to better illustrate the concepts taught. For instance: pictures, objects, videos, board, etc. are shown/used during the sessions. As suggested in [4], using different sources of information (visual, verbal, sound, etc.) promotes the use of different learning strategies, which impacts on the learning effectiveness.

- Music: it is an important component used during the sessions. On the one hand it is used to guide the dynamics of the group and their movements (for instance, during warm-up, faster rhythms are used, while during exploration, slow music is played), and on the other hand, it serves as motivational support (specially to start the sessions and breaking the ice). If children like it, they would easily engage in the task and would start moving around along with the teacher. Similar findings were reported in [14].

- Performance and appreciation: Pupils like showing the knowledge acquired and their own creations to receive approval from the teacher. Their motivation increases and they feel confident to be challenged with new things. Moreover, it serves as a brainstorming process where their creativity is stimulated by observing others' creations.

- Motivational feedback: Fear of failure may restrain creative thinking [11]. The teacher constantly tells the pupils how well they are doing. This encourages to continue the task. When negative feedback should be given, positive words are used while phrasing her comments to avoid disappointment.

- Activity summary: At the beginning of each session the teacher would briefly explain the goal and content of the session taking place. If possible, she will also recapitulate what they did in previous sessions to link the concepts worked. During the session, she would briefly describe the next activity to perform so the pupils are aware of what is coming next. At the end of session, before debriefing, she summarizes the lesson they took part of.

\subsection{General Methodology}

The general methodology distilled from the observations of these sessions is composed of the following stages:

1. Warm-up: the teacher starts the session with slow and smooth movements, to stretch and strengthen the body. She gradually increases the dynamics of the movements to completely warm the body.

2. Exploration: the teacher explains movement concepts while practicing them with the pupils.

3. Creation: individually or in groups, pupils create dance phrases (a sequence of movements) based on the concepts learned in the exploration stage.

4. Performance and Appreciation: pupils perform the dance phrases created during the session to show their work to the rest of the classmates. This stage enables them to discuss about the different performances identifying and reinforcing the learned concepts. 


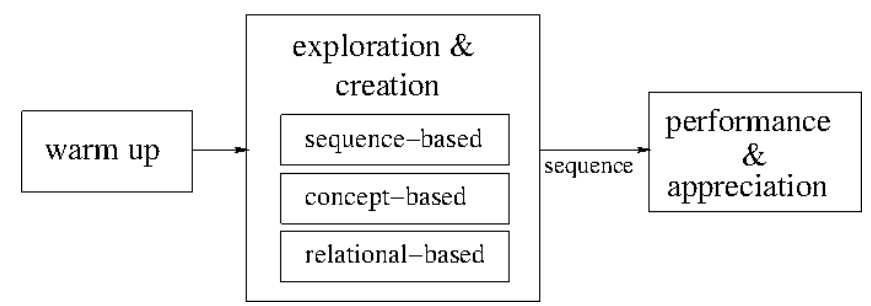

Fig. 2: General methodology for creative dance. The exploration and creation stages are coupled based in three learning models.

Stages 2 through 4 are repeated several times during a session. At each cycle a different concept is explained, explored and performed/observed.

Each session tackles different concepts, and therefore, the stages may vary from one session to another one, extending more when required. However, the methodology remains the same. Fig. 2 depicts the overall methodology. We will next go through the different models.

\subsection{Learning Models}

Through the sessions we took part of, several methods were used to teach dance. While the outcome is the same in all models, i.e. the creation and performance of a sequence, the process through which the pupils learn and create the individual movements of the sequence differ. In this work we focus on three different methodologies to apply in the design of a robot dance tutor.

Sequence-based Model. This model corresponds to a more traditional one, where the goal is to teach a specific sequence of movements. The sequences is created by the teacher and the pupils reproduce it the in the same way. Fig. 3 depicts the model. Each step (or movement) is shown one at a time to evaluate the performance of the pupils. Once a step is taught, it is added to the sequence. The expanded sequence is practiced every time a new step is added.

This model does not require the use of creativity from the pupils point of view. It is usually used during the warm up stage and in the first session when the teacher and the pupils meet for the first time. However, to introduce creativity in the process, variations on the sequence learned can be applied. The following steps gradually motivate pupils to introduce their own creations:

1. Modify the order of the movements in the predefined sequence.

2. Include repetitions of some movements through the sequence.

3. Modify some movements in terms of dynamics (faster, slower), levels (on the floor), or spatial representation (mirroring).

4. Include as many new movements as they desired.

Thus, although pupils start copying a set of movements, they can end up with a completely different sequence according to their own creativity. 


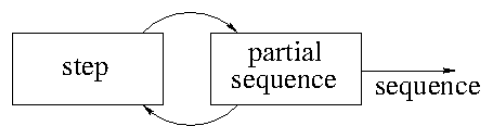

Fig. 3: Diagram for the sequence-based learning model.

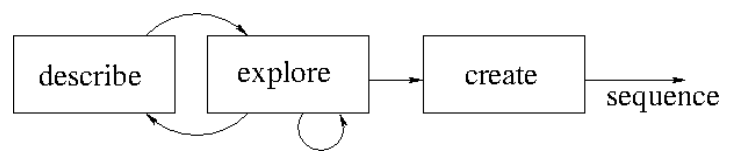

Fig. 4: Diagram for both concept and relational-based models.

Concept-based Model. This model allows the introduction of the foundations of dance, i.e. movement concepts, along with the exploration of the body movement (Fig. 4). First a a concept is described through different means, i.e. performing a movement, showing an image, describing it verbally, through analogies. Next, the pupils explore the new idea using their own body. They are free to create any variation they can think of as long as it corresponds to the concept described. The process is repeated for different concepts until the ideas are well understood. Finally, the pupils are asked to create a sequence with the concepts learned. They can either create their own sequence as they like, or a guideline can be provided (a sequence of concepts), but it is up to the pupils to select the movements used to represent the concepts in the sequence. Fig. 5 shows some of the concepts taught during the dance sessions we took part of.

Relational-based Model. In Section 2 we stated that creative dance can be used to convey concepts of specific themes linked to movement concepts. Through this model, the pupils not only reinforce their knowledge on the movement concepts seen so far, but also learn and establish links with other ideas. The model can be applied bidirectionally: departing from a theme concept and linking it with movement concepts, or the other way around, i.e. based on a movement concept, link it to theme concepts. Either way, first a description of the movement (or theme concept) is given (describe stage in Fig. 4) and the pupils explore different theme concepts (or movements) linked to that description.

For example, to link from a theme concept to a movement concept, the teacher shows an image of a fruit (say a banana) and asks the pupils which (movement) shapes they can see in a banana. A plausible answer would be curved shape. On the other sense, from movement concept to theme concept, the teacher would name a concept (say, spiky shape), and the pupils should think of a fruit which matches that concept and then create a movement with their body to reproduce it (in this case, a valid solution would be a pineapple). 

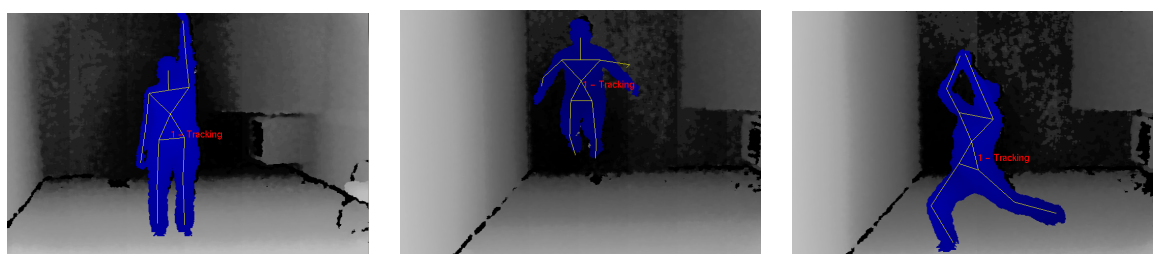

Fig. 5: Examples of movement concept used in the concept-based learning model. From left to right: straight shape (matching the shape of green onions), spring (related to simple carbohydrates, which allow us to perform quick actions) and pull (related to proteins, wich provide energy for strong actions).

To consolidate the concepts linked, the pupils create a sequence as in the previous model, i.e. either on their own or based on a given sequence of theme or movement concepts.

\section{Designing a Dance Robot Tutor}

The robot's behavior is twofold. On the one hand to perform a dance activity where the robot acts as a tutor guiding the child through the different stages. And on the other hand, to keep the child engaged in the task as much as possible, not only to finish the task, but to repeat the encounter in future occasions.

To this end, the methodology and models described in the previous section have been implemented. Moreover, based on our previous work [12], additional cues for enhancing the social interaction are also included in the design of the robot behavior. This type of behaviors prevent the robot of being too static, being more human-like as suggested in the literature [3] [16] [8] [15]. In this work we make use of the following:

- head movements: the head randomly moves in a smooth way. When the robot addresses a direct question to the child, it faces the child to emphasize that the robot is expecting the child to respond.

- blinking eyes: the robot's eyes are provided with colored LEDs. Turning them on and off simulates natural human eye blinking making the robot appearance more "alive".

- spatial orientation: while interacting with humans, it is important to maintain an appropriate spatial disposition while executing a task (a spatialorientational arrangement [9]). In this work the robot should be facing the user to show the different motions. Due to the movement of the robot while performing the dance motions, the robot may end up facing some other place in the room instead of facing the user.

- name reference: the robot randomly refers to the child by its name to reinforce the bond between them throughout the session. 


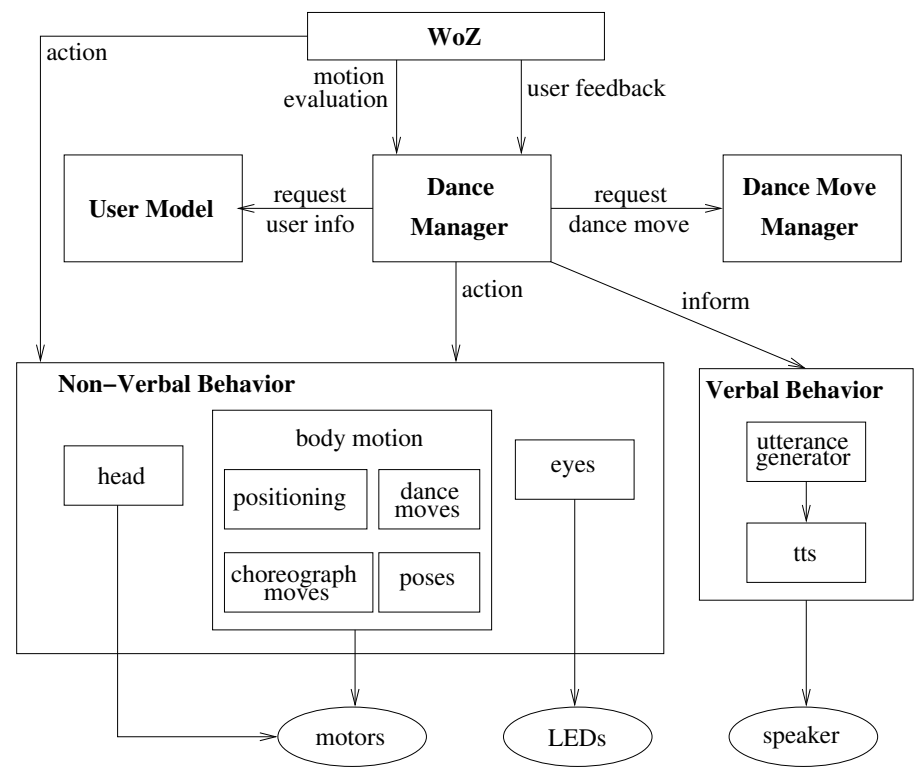

Fig. 6: Diagram of the system architecture. Arrows represent the flow of information between components.

We next briefly describe the system architecture used in this work. An overall description is illustrated in Fig. 6. The implementation of the system has been done using $\mathrm{Urbi}^{2}$, an open-source software platform to control robots.

- Dance Manager: controls the overall robot behavior, implementing both the methodologies described in Sec. 2.3 and the above engaging behaviors.

- WoZ: provides external input while the perceptual components are completed. Allows to introduce the child's performance evaluation and answers to questions. Additionally, it allows the operator to re-position the robot.

- Dance Move Manager: manages the library of dance movements and provides information to the Non-Verbal Behavior for the execution of those.

- User Model: in charge of handling the users' interaction history to enable adaptation in the decision process. Besides storing general information about the child (such as Id, name, age and gender), it also keeps track of the dance moves performed

- Verbal Behavior: responsible for the verbal output of the robot. The utterances are based on canned text manually created. Variations of each message have been produced to avoid repeated verbalization.

- Text-To-Speech: in charge of the speech synthesis, we use the commercial Acapella TTS [6] built-in the robot.

- Non-Verbal Behavior: responsible for the non-verbal output of the robot.

${ }^{2}$ www.urbiforge.org 


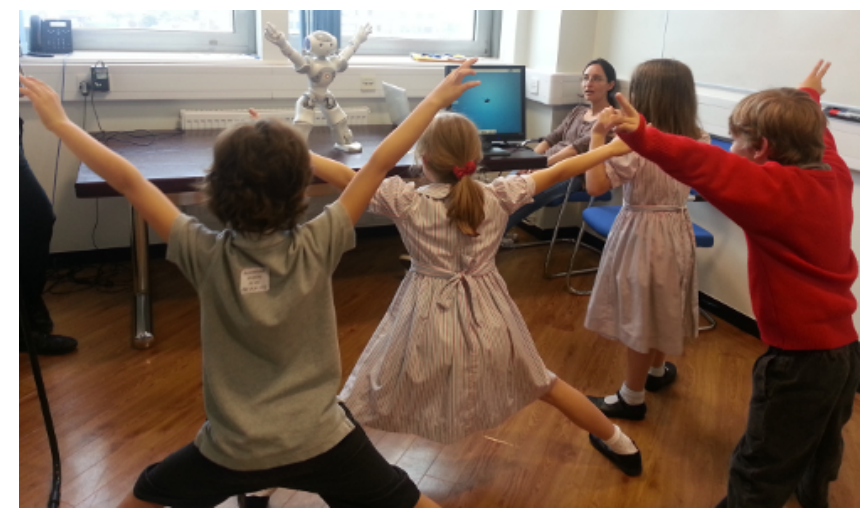

Fig. 7: Snapshot of the pilot test. Children interacted with the robot in groups of four. In the image, they are performing a big shape.

- Body Motion: manages the robot's body motion.

- Blinking Eyes: controls the LEDs located in the robot eyes to emulate blinking eyes.

- Head Motion: controls the head movement when no other motion occurs.

\section{Preliminary Results}

The robot used is the Nao, a humanoid robot from Aldebaran Robotics. ${ }^{3}$ The Nao is almost $58 \mathrm{~cm}$ tall, weights $5.2 \mathrm{~kg}$ and has a cartoon-like appearance which was considered especially suitable for use with children. It has 25 degrees of freedom, which allow smooth motion specially required for dance movements.

Tables 1 and 2 describe a trial example of a session. The dance topic corresponds to shapes and it is linked to nutrition through fruits and vegetables.

A pilot test of the first prototype has been carried out. In the test, 17 children divided in four groups between 8 and 9-years-old had a chance to interact with the robot (Fig. 7). Although the system is intended to be evaluated later on in one-to-one interactions, we believe that this initial feedback on the proposed framework is valuable to further improve our work.

In general children responded in a very positive way. They liked the robot and the way it moved. They engaged with the robot copying or creating movements and they understood the movement concepts. Creativity easily emerged and they expected the robot to repeat their own creations in turn. They addressed the robot in different ways, verbally (making questions) and non-verbally (waving hands) demonstrating interest in interacting with it.

They reported that sometimes it was difficult to understand what the robot said and that the instructions were not too clear so they felt slightly lost at some

\footnotetext{
${ }^{3}$ www.aldebaran-robotics.com
} 
points. They also expected the robot to perform some well know dances (e.g. most of them asked for the Gangnam Style).

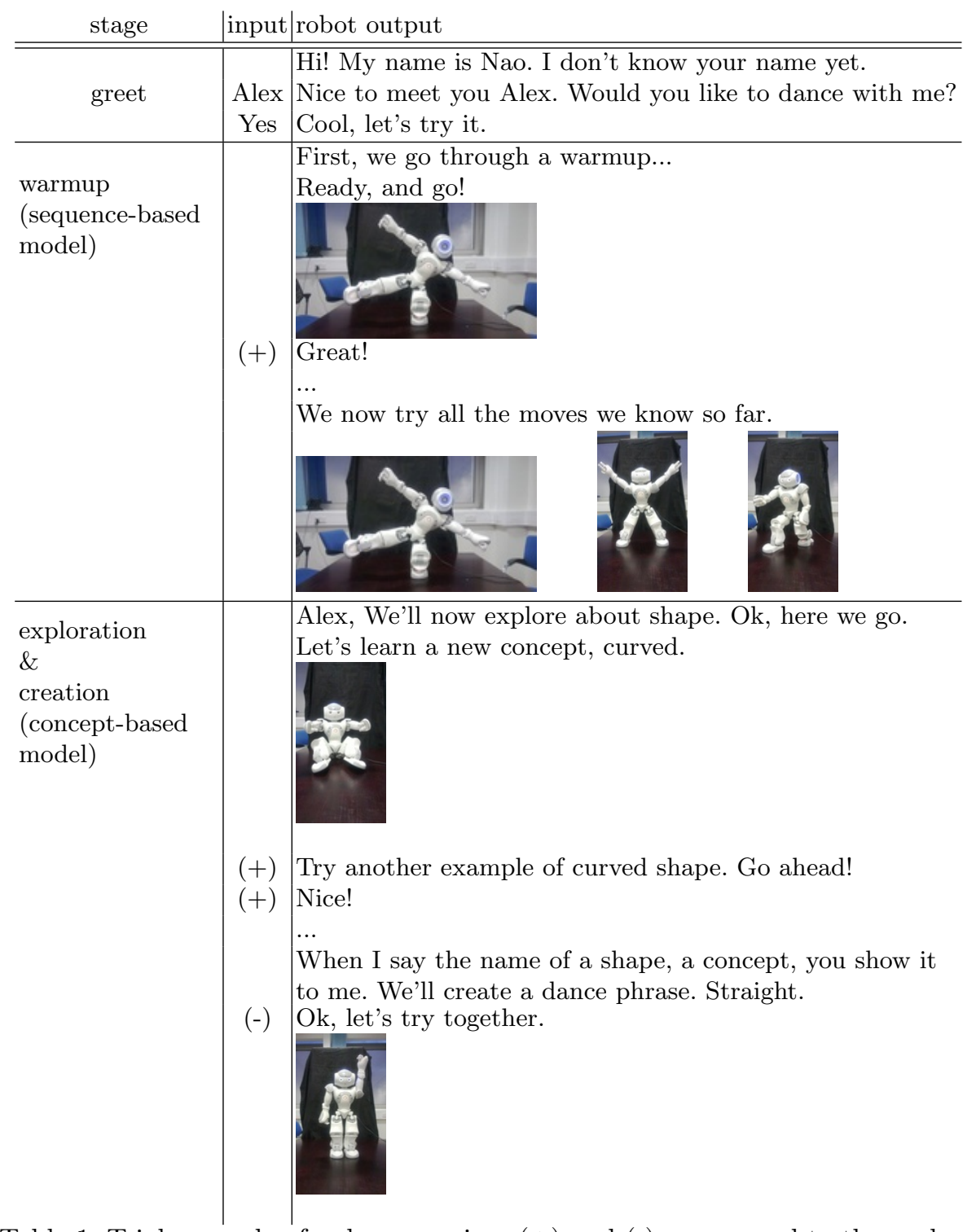

Table 1: Trial example of a dance session. (+) and (-) correspond to the evaluation of the child's performance. 


\begin{tabular}{|c|c|c|}
\hline stage & put & bot output \\
\hline $\begin{array}{l}\text { healthy diet } \\
\text { overview. }\end{array}$ & & $\begin{array}{l}\text { Vegetables and fruits provide vitamins and minerals to } \\
\text { our body important to prevent from diseases... }\end{array}$ \\
\hline $\begin{array}{l}\text { exploration } \\
\& \\
\text { creation } \\
\text { (relational- } \\
\text { based model) } \\
\text { from theme } \\
\text { to movement } \\
\text { concept }\end{array}$ & $\begin{array}{c}(-) \\
\text { curved }\end{array}$ & $\begin{array}{l}\text { Let's link the concepts seen so far with the images in } \\
\text { the screen. Tell me which shapes can you see. }\end{array}$ \\
\hline $\begin{array}{l}\text { exploration } \\
\& \\
\text { creation } \\
\text { (relational- } \\
\text { based model) } \\
\text { from movement } \\
\text { concept to theme }\end{array}$ & $(-)$ & $\begin{array}{l}\text { 'Il the name of a shape and you have to show me a fruit } \\
\text { or vegetable that has that concept. Spiky } \\
\text { Ok, let's try together. Spiky like a pineapple. } \\
\text { ro }\end{array}$ \\
\hline farewell & & cing with you! Thanks. \\
\hline
\end{tabular}

Table 2: (continuation of Table 1) Trial example of a dance session. (+) and (-) correspond to the evaluation of the child's performance.

\section{Conclusions}

We have proposed an approach for creative dance to study child-robot interaction through several encounters. We took part of dance sessions in order to extract strategies and models to inspire and justify the design of a robot dance tutor.

In the near future we plan to evaluate our system with children in one-to-one interactions during three sessions. Based on our previous work, the evaluation of the interaction will be based on video coding schemes to analyze the level of interaction. Regarding the impact of creativity, we plan to look at the outcomes generated by the children to assess whether creativity emerged through the interactions and how it influenced on the interaction itself. Moreover, we will be able to evaluate whether the interactions have an impact on knowledge gain.

\section{References}

1. Physical Education, The National Curriculum for England, 2011.

2. N Bianchi-Berthouze. Understanding the role of body movement in player engagement. Human-Computer Interaction, 28(1):42-75, 2013.

3. A Bruce, I Nourbakhsh, and R Simmons. The role of expressiveness and attention in human-robot interaction. In Int. Conf. on Robotics and Automation, volume 4, pages 4138-4142, 2002. 
4. M Cadopi, J. F. Chatillon, and R. Baldy. Representation and performance: Reproduction of form and quality of movement in dance by eight- and 11-year-old novices. British Journal of Psychology, 86:217-225, 1995.

5. M. Csíkszentmihályi. Creativity: flow and the psychology of discovery and invention. HarperCollinsPublishers, 1996.

6. D Gouaillier, V Hugel, P Blazevic, C Kilner, J Monceaux, P Lafourcade, B Marnier, J Serre, and B Maisonnier. The nao humanoid: a combination of performance and affordability. CoRR, abs/0807.3223, 2008.

7. E Hui, B Tsan keung Chui, and J Woo. Effects of dance on physical and psychological well-being in older persons. Archives of Gerontology and Geriatrics, 49:e45-e50, 2009.

8. T Kanda, R Sato, N Saiwaki, and H Ishiguro. A two-month field trial in an elementary school for long-term human-robot interaction. Transactions on Robotics, 23(5):962-971, 2007.

9. A Kendon. Spacing and orientation in co-present interaction. In Development of Multimodal Interfaces: Active Listening and Synchrony, volume 5967 of LNCS, pages 1-15. 2010.

10. M Michalowski, R Simmons, and H Kozima. Rhythmic attention in child-robot dance play. In Int. Symposium on Robot and Human Interactive Communication, pages 816-821, 2009.

11. R S Nickerson. 20 enhancing creativity. Handbook of creativity, page 392, 1999.

12. R Ros, I Baroni, and Y Demris. Adaptive human robot interaction in sensorimotor task instruction: from human to robot dance tutors. Submitted, May 2013.

13. I Von Rossberg-Gempton, J Dickinson, and G Poole. Creative dance: Potentiality for enhancing social functioning in frail seniors and young children. The Arts in Psychotherapy, 26:313-327, 1999.

14. F Tanaka, J Movellan, B Fortenberry, and K Aisaka. Daily HRI evaluation at a classroom environment: reports from dance interaction experiments. In Conf. on Human-robot interaction, pages 3-9, 2006.

15. Fumihide Tanaka and Shizuko Matsuzoe. Children teach a care-receiving robot to promote their learning: Field experiments at a classroom for vocabulary learning. Journal of Human-Robot Interaction, 1(1):78-95, 2012.

16. Y. Yoshikawa, K. Shinozawa, H. Ishiguro, N. Hagita, and N. Miyamoto. The effects of responsive eye movement and blinking behavior in a communication robot. In Int. Conf. on Intelligent Robots and Systems, pages 4564-4569, 2006. 